\title{
CRESCIMENTO DE MUDAS DE ANGICO-VERMELHO (Anadenanthera macrocarpa (Benth.) Brenan) SOB DIFERENTES DOSES DE MACRONUTRIENTES ${ }^{1}$
}

Elzimar de Oliveira Gonçalves ${ }^{2}$, Haroldo Nogueira de Paiva ${ }^{3}$, Julio Cesar Lima Neves ${ }^{4}$ e José Mauro Gomes ${ }^{3}$

\begin{abstract}
RESUMO - Neste trabalho, objetivou-se avaliar, por meio de características morfológicas, o efeito de doses de macronutrientes no crescimento e desenvolvimento de mudas de angico- vermelho (Anadenanthera macrocarpa (Benth.) Brenan) produzidas em amostras de três classes de solo (Argissolo Vermelho-Amarelo, Latossolo Vermelho-Amarelo álico e Latossolo Vermelho-Amarelo distrófico). As mudas foram plantadas em vasos com capacidade para $2,1 \mathrm{dm}^{3}$. O experimento foi conduzido no período de dezembro de 2004 a maio de 2005. Os tratamentos foram delimitados segundo uma matriz baconiana, em que se variaram os macronutrientes em três doses diferentes, mais dois tratamentos adicionais (zero e base), com quatro repetições. As fontes de N, K e S foram parceladas em quatro vezes (0-30-60-90 dias). Em termos gerais, o maior crescimento das plantas ocorreu no Latossolo Vermelho-Amarelo álico. O nutriente que mais surtiu efeitos significativos foi o $\mathrm{P}$, sendo recomendadas doses de 150 a $250 \mathrm{mg} \mathrm{dm}^{-3}$. Para a aplicação de $\mathrm{N}$, recomendam-se doses mínimas de $50 \mathrm{mg} / \mathrm{dm}^{3}$. A aplicação de $\mathrm{S}$ deve ser de $20 \mathrm{a} 80 \mathrm{mg} \mathrm{dm}^{-3}$. As respostas à aplicação dos demais nutrientes (Ca, Mg e K), em muitos casos, não ocorreu, evidenciando-se que a espécie tem baixo requerimento por eles. Entretanto, partindo desse estudo, sugerem-se novos estudos com os nutrientes $\mathrm{K} \mathrm{e} \mathrm{Ca}$, para produção de mudas da referida espécie nesses solos.
\end{abstract}

Palavras-chave: Angico-vermelho, macronutrientes e produção de mudas.

\section{GROWTH OF ANGICO VERMELHO (Anadenanthera macrocarpa (Benth.) Brenan) SEEDLINGS UNDER DIFFERENT MACRONUTRIENT DOSES}

\begin{abstract}
This work aimed to evaluate, by means of morphological characteristics, the effect of macronutrient doses on the growth of angico vermelho (Anadenanthera macrocarpa (Benth.) Brenan) seedlings cultivated in three soils (Red Yellow Argisol, Red Yellow Dystrophic Latosol and Red Yellow alic Latosol). The seedling were sown in vases with capacity of $2.1 \mathrm{dm}^{3}$. The experiment was carried out from December 2004 to June 2005. The treatments were delimited according to a Baconian matrix, with macronutrients varying in three different doses, plus two additional treatments (zero and base), with four repetitions. The sources of $N, K$ and $S$ were applied in four times (0-30-60-90 days). In general, the highest plant growth occurred in the Red Yellow alic Latosol soil. The nutrient presenting the most significant effects was $P$, with doses of 150$250 \mathrm{mg} \mathrm{dm}^{-3}$ being recommended. For $\mathrm{N}$, minimum doses of $50 \mathrm{mg} / \mathrm{dm}^{3}$ are recommended. The nutrient $S$ must be applied between 20 and $80 \mathrm{mg} \mathrm{dm}^{-3}$. The responses to the other nutrients (Ca, $\mathrm{Mg}$ and $\mathrm{K}$ ), in many cases did not occur, suggesting that the species has low requirement for them. However, based on this work, further studies are suggested on the nutrients $K$ and $C a$, for production of seedlings of the studied species in these soils.
\end{abstract}

Keywords: Angico-vermelho, macronutrients and seedling production .

\footnotetext{
${ }^{1}$ Recebido em 18.06.2007 e aceito para publicação em 22.08.2008.

${ }^{2}$ Programa de Pós-Graduação em Ciência Florestal da Universidade Federal de Viçosa (UFV). E-mail: <euzzy @ yahoo.com>.

${ }^{3}$ Departamento de Engenharia Florestal da UFV. E-mail: <hnpaiva@ufv.br>.

${ }^{4}$ Departamento de Solos da UFV.
} 


\section{INTRODUÇÃO}

O angico-vermelho é uma árvore da família Mimosaceae e apresenta expressiva regeneração natural, ocorrendo indiferentemente em solos secos e úmidos; é tolerante a solos rasos, compactados, mal drenados e até encharcados, de textura média a argilosa. Apresenta crescimento de moderado a rápido, podendo atingir, quando em ótimas condições, produtividades de até 25,55 $\mathrm{m}^{3}$. ha ${ }^{-1}$.ano (CARVALHO, 2003). De acordo Lorenzi (2000), a característica de rápido crescimento a torna interessante para ser aproveitada em reflorestamentos de áreas degradadas. A espécie possui, ainda, outras utilidades, servindo para construção civil, produção de carvão etc. (LORENZI, 2000).

Devido a essas potencialidades, estudos relacionados à sua fertilização têm sido realizados, visando à melhoria da qualidade das mudas produzidas, o que aumentaria as suas chances de sucesso na implantação no campo. Entre esses estudos, citamse os de Schumacher et al. (2004), que verificaram que a espécie é responsiva à aplicação de $\mathrm{P}$, apresentando os maiores valores de crescimento quando aplicados $450 \mathrm{mg}$ de $\mathrm{P} \mathrm{dm}^{-3}$. Bernardino et al. (2005) concluíram que as características morfológicas da espécie não foram influenciadas pelo aumento de saturação por bases quando as mudas foram cultivadas em um Argissolo; todavia, em Latossolos (distrófico e álico) o aumento da saturação por bases implicou mudas de melhor qualidade. Em relação à aplicação de $\mathrm{N}$ e ao seu efeito sobre o crescimento do angico-vermelho, Marques et al. (2006) recomendaram que, na produção de mudas de angico-vermelho, a dose de $180 \mathrm{mg} \mathrm{dm}^{-}$ ${ }^{3}$ de N, tendo como fonte o sulfato de amônio, é a que garante muda de melhor qualidade.

Apesar de esses estudos já possibilitarem indicação mais acertada da fertilização para a produção das mudas, pelo menos para o $\mathrm{P}, \mathrm{N}$, Ca e $\mathrm{Mg}$, faltam estudos de outros nutrientes, bem como o comportamento conjunto da aplicação de todos eles. Logo, o objetivo deste trabalho foi determinar a dose de cada macronutriente que fará com que as mudas tenham maior crescimento e desenvolvimento.

\section{MATERIAL E MÉTODOS}

O experimento foi conduzido em casa de vegetação, no Viveiro de Pesquisas Florestais do Departamento de Engenharia Florestal da Universidade Federal de Viçosa, em Viçosa, Minas Gerais, no período de dezembro de 2004 a maio de 2005, com temperatura média diária de $21,11^{\circ} \mathrm{C}$, média das máximas de $26,52^{\circ} \mathrm{C}$ e média das mínimas de $17,68^{\circ} \mathrm{C}$. As médias diárias de precipitação e umidade relativa do ar foram de 7,5 $\mathrm{mm}$ e 76,66\%, respectivamente. As amostras dos solos utilizados como substrato na produção das mudas foram retiradas cerca de 0,30 $\mathrm{m}$ abaixo da camada superficial de três classes de solos, Argissolo Vermelho-Amarelo (PVA), Latossolo Vermelho-Amarelo álico (LVA) e Latossolo VermelhoAmarelo distrófico (LVD), predominantes na região da Zona da Mata de Minas Gerais, de acordo com Resende et al. (1988), das quais foram determinadas as características físicas e químicas (Quadro 1). As amostras foram secas ao ar e passadas em peneiras de malha de $5 \mathrm{~mm}$. Em seguida, pesaram-se 2,1 kg de cada solo, que foram colocados em embalagens plásticas de capacidade aproximada de $5 \mathrm{~kg}$. Posteriormente, os sais fornecedores de cálcio e magnésio $\left(\mathrm{CaCO}_{3} \mathrm{e}\right.$ $\mathrm{MgCO}_{3}$ ) foram adicionados, nas quantidades definidas pelos tratamentos do Quadro 2, e homogeneizados. Posteriormente, adicionou-se água até a capacidade de campo, e as amostras permaneceram, para reação do calcário, por 30 dias, com manutenção do teor de umidade.

Quadro 1 - Atributos químicos das amostras de solos utilizadas na produção das mudas antes da aplicação dos tratamentos Table 1 -Chemical attributes of the samples used in the production of the seedlings before application of the treatments

\begin{tabular}{|c|c|c|c|c|c|c|c|c|c|c|c|}
\hline \multirow[t]{2}{*}{ Solo } & \multirow{2}{*}{$\frac{\mathrm{pH}}{\mathrm{H}_{2} \mathrm{O}}$} & $\mathrm{P}$ & $\mathrm{K}$ & $\mathrm{Ca}^{2+}$ & $\mathrm{Mg}^{2+}$ & $\mathrm{Al}^{3+}$ & $\mathrm{H}+\mathrm{Al}$ & SB & $(\mathrm{T})$ & $\mathrm{V}$ & $\mathrm{m}$ \\
\hline & & \multicolumn{2}{|c|}{$\mathrm{mg} \mathrm{dm}^{-3}$} & \multicolumn{6}{|c|}{$\mathrm{cmol}_{\mathrm{c}} \mathrm{dm}^{-3}$} & \multicolumn{2}{|c|}{$\%$} \\
\hline$\overline{\mathrm{PVA}}$ & 6,00 & 2,10 & 14 & 1,60 & 0,10 & 0,00 & 3,63 & 1,74 & 5,37 & 32 & 0 \\
\hline LVA & 4,80 & 1,40 & 32 & 0,20 & 0,00 & 0,80 & 3,96 & 0,28 & 4,24 & 7 & 74 \\
\hline LVD & 5,20 & 0,8 & 49 & 0,30 & 0,00 & 0,00 & 1,82 & 0,43 & 2,25 & 19 & 0 \\
\hline
\end{tabular}

pH em água, $\mathrm{KCl}$ e $\mathrm{CaCl}_{2}$ - Relação 1: 2,5, P e K - Extrator Mehlich 1, $\mathrm{Ca}^{2+}, \mathrm{Mg}^{2+}$ e $\mathrm{Al}^{3+}-\mathrm{Extrator}: \mathrm{KCl} 1 \mathrm{~mol} / \mathrm{L}, \mathrm{H}+\mathrm{Al}-\mathrm{Extrator}$ Acetato de Cálcio $0,5 \mathrm{~mol} / \mathrm{L}-\mathrm{pH} 7,0$.

R. Árvore, Viçosa-MG, v.32, n.6, p.1029-1040, 2008 
Quadro 2 - Descrição dos tratamentos Table 2 -Description of the treatments

\begin{tabular}{|c|c|c|c|}
\hline Tratamentos & Descrição & Tratamentos & Descrição \\
\hline 1 & Referência (*) & 11 & $\mathrm{~K}=200 \mathrm{mg} \mathrm{dm}-^{3}$ \\
\hline 2 & $\operatorname{Dose}=0(* *)$ & 12 & $\mathrm{Ca}=0,8 \mathrm{cmol}_{\mathrm{c}} \mathrm{dm}^{-3}$ \\
\hline 3 & $\mathrm{~N}=50 \mathrm{mg} \mathrm{dm-3}$ & 13 & $\mathrm{Ca}=1,2 \mathrm{cmol}_{\mathrm{c}} \mathrm{dm}^{-3}$ \\
\hline 4 & $\mathrm{~N}=150 \mathrm{mg} \mathrm{dm}^{-3}$ & 14 & $\mathrm{Ca}=1,4 \mathrm{cmol}_{\mathrm{c}} \mathrm{dm}^{-3}$ \\
\hline 5 & $\mathrm{~N}=200 \mathrm{mg} \mathrm{dm}-^{3}$ & 15 & $\mathrm{Mg}=0,2 \mathrm{cmol}_{\mathrm{c}} \mathrm{dm}^{-3}$ \\
\hline 6 & $\mathrm{P}=150 \mathrm{mg} \mathrm{dm}-^{3}$ & 16 & $\mathrm{Mg}=0,6 \mathrm{cmol}_{\mathrm{c}} \mathrm{dm}^{-3}$ \\
\hline 7 & $\mathrm{P}=450 \mathrm{mg} \mathrm{dm}^{-3}$ & 17 & $\mathrm{Mg}=0,8 \mathrm{cmol}_{\mathrm{c}} \mathrm{dm}^{-3}$ \\
\hline 8 & $\mathrm{P}=600 \mathrm{mg} \mathrm{dm}-^{3}$ & 18 & $\mathrm{~S}=20 \mathrm{mg} \mathrm{dm}^{-3}$ \\
\hline 9 & $\mathrm{~K}=50 \mathrm{mg} \mathrm{dm}-^{3}$ & 19 & $\mathrm{~S}=60 \mathrm{mg} \mathrm{dm}-^{3}$ \\
\hline 10 & $\mathrm{~K}=150 \mathrm{mg} \mathrm{dm}^{-3}$ & 20 & $\mathrm{~S}=80 \mathrm{mg} \mathrm{dm}^{-3}$ \\
\hline
\end{tabular}

(*) $\mathrm{N}=100 \mathrm{mg} / \mathrm{dm}^{3}, \mathrm{P}=300 \mathrm{mg} / \mathrm{dm}^{3}, \mathrm{~K}=100 \mathrm{mg} / \mathrm{dm}^{3} \mathrm{Ca}=1 \mathrm{cmol} / \mathrm{dm}^{3} \mathrm{Mg}=0,4 \mathrm{cmol} / \mathrm{dm}^{3} \mathrm{~S}=40 \mathrm{mg} / \mathrm{dm}^{3}$ (**) Solo sem adição de nutrientes.

Decorridos os 30 dias, os solos receberam adubação com os demais nutrientes, de acordo com as quantidades definidas nos tratamentos do Quadro 2, sendo o N, o K e o $\mathrm{S}$ parcelados em quatro vezes $(0,30,60,90$ dias) após a semeadura. Os sais utilizados para a adubação foram: $\mathrm{NH}_{2} \mathrm{PO}_{4} \cdot \mathrm{H}_{2} \mathrm{O}$ para o $\mathrm{Ne} \mathrm{P}, \mathrm{NH}_{4} \mathrm{NO}_{3}$ para o N, $\left(\mathrm{NH}_{4}\right)_{2} \mathrm{SO}_{4}$ para o N e S; $\mathrm{K}_{2} \mathrm{SO}_{4}$ para $\mathrm{S}$ e $\mathrm{K}$; e $\mathrm{KCl}$ para $\mathrm{K}$.

Adicionou-se também uma solução de macronutrientes, nas seguintes doses: $\mathrm{B}=0,81 \mathrm{mg}$ $\mathrm{dm}^{-3}\left(\mathrm{H}_{3} \mathrm{BO}_{3}\right), \mathrm{Cu}=1,33 \mathrm{mg} \mathrm{dm}^{-3}\left(\mathrm{CuSO}_{4} .5 \mathrm{H}_{2} \mathrm{O}\right), \mathrm{Mo}$ $=0,15 \mathrm{mg} \mathrm{dm}^{-3}\left[\left(\mathrm{NH}_{4}\right)_{6} \mathrm{Mo}_{7} \mathrm{O}_{24} \cdot 4 \mathrm{H}_{2} \mathrm{O}\right], \mathrm{Mn}=3,66 \mathrm{mg}$ $\mathrm{dm}^{-3}\left(\mathrm{MnCl}_{2} \cdot \mathrm{H}_{2} \mathrm{O}\right)$ e $\mathrm{Zn}=4,0 \mathrm{mg} \mathrm{dm}^{-3}\left(\mathrm{ZnSO}_{4} \cdot 7 \mathrm{H}_{2} \mathrm{O}\right)$ (ALVAREZ V., 1974). Após a adubação e em virtude da homogeneização, os solos foram acondicionados aos vasos.

Os tratamentos foram obtidos segundo uma matriz baconiana (TURRENT, 1979), em que se avaliaram seis nutrientes (N, P, K, Ca, Mg e S) em três diferentes doses, $\mathrm{e}$, ainda, dois tratamentos adicionais, sendo um com doses de referência e outro sem adição de nutrientes, totalizando 20 tratamentos (Quadro 2), que foram dispostos em delineamento de blocos inteiramente casualizados, com quatro repetições.

A parcela experimental foi constituída por um vaso de polipropileno rígido, contendo cada um $2,1 \mathrm{dm}^{3} \mathrm{de}$ solo e uma muda.

As sementes de angico-vermelho foram adquiridas no Setor de Silvicultura do DEF/UFV e inoculadas com estirpes selecionadas de Bradyrhizobium, fornecidas pelo Centro Nacional de Pesquisa em Agrobiologia/ EMBRAPA, Seropédica, RJ. Cada vaso plástico recebeu
10 sementes, efetuando-se o primeiro desbaste aos 15 dias após a emergência, deixando-se duas plantas por vaso. Após 30 dias da semeadura, um segundo desbaste foi realizado, deixando-se apenas uma planta por vaso.

Durante o período experimental, o teor de umidade do solo foi mantido próximo de $60 \%$ da capacidade de campo, procedendo-se a um monitoramento diário para esse controle.

Medições de altura e diâmetro do coleto das mudas foram realizadas 120 dias após a semeadura. Em seguida, as plantas foram colhidas e subdivididas em raízes e parte aérea (caule e folhas), lavadas em água destilada e postas para secar em estufa a $45^{\circ} \mathrm{C}$, com circulação forçada de ar até peso constante. Depois de secas, foram pesadas em balança analítica com precisão de $0,01 \mathrm{~g}$, para determinação da matéria seca da parte aérea (PMSPA) e matéria seca da raiz (PMSR), obtendo-se pelo somatório das duas o peso da matéria seca total (PMST).

Os dados foram interpretados estatisticamente, por meio de contrastes, análises de variância e regressão, utilizando-se o programa SAEG (Sistema de Análises Estatística e Genética) (EUCLYDES, 1997) e o Statistica.

Testou-se o efeito da adição de macronutrientes versus a não-adição, através de um contraste entre as médias do tratamento $2($ dose $=0)$ versus as médias dos demais tratamentos $(\mathrm{C}=\mathrm{T} 1-19 \mathrm{~T} 2+\mathrm{T} 3+\mathrm{T} 4+\ldots+\mathrm{T} 19+\mathrm{T} 20)$.

Para obtenção das equações, utilizaram-se quatro pontos, sendo três referentes às doses testadas para cada nutriente e um referente ao tratamento adicional de referência, que foi empregado em para todos os nutrientes.

R. Árvore, Viçosa-MG, v.32, n.6, p.1029-1040, 2008 
As curvas de respostas à adição de doses de cada nutriente resultaram de modelos de regressão, nos quais os coeficientes foram testados com base nos valores do quadrado médio do resíduo da ANOVA conjunta, analisada a $1 \%, 5 \%$ e $10 \%$ de probabilidade. Adicionalmente, observaram-se o $\mathrm{R}^{2}$, a significância dos betas da equação e o significado biológico dos modelos.

A partir das equações, no caso de modelos quadráticos e de raiz quadrada, foram determinados as doses recomendadas de nitrogênio, fósforo, potássio, enxofre, cálcio e magnésio, para obtenção de $90 \%$ dos valores máximos estimados com relação às diversas características estudadas.

\section{RESULTADOS E DISCUSSÕES}

O contraste entre os tratamentos com fertilizações versus sem fertilização (dose $=0$ ), indicou que os tratamentos com adição de sais foram significativamente superiores (Quadro 3), ou seja, a aplicação de fertilizantes que continham $\mathrm{N}, \mathrm{P}, \mathrm{K}, \mathrm{Ca}, \mathrm{Mg}$ e $\mathrm{S}$ possibilitou significativo aumento do crescimento das mudas de angico-vermelho nas três classes de solos.

As baixas médias de crescimento observadas em todas as características estudadas no tratamento sem adição de sais eram esperadas, pois os nutrientes exercem diversas funções na planta; logo, a sua omissão irá comprometer diversos processos metabólicos, o que implica diminuição do crescimento e desenvolvimento das plantas.

\subsection{Efeito do Nitrogênio}

Os valores médios observados nas diversas características avaliadas em geral aumentaram, em razão da elevação de doses de N (Quadro 4). À exceção do PMSR, aplicação de doses crescentes de N provocou efeitos que resultaram em modelos linear, hiperbólico e quadrático, de acordo com o solo em questão (Quadro 5).

O efeito da aplicação de $\mathrm{N}$ foi linear positivo para o diâmetro, PMSPAe PMST no substrato LVA, indicando que, para essas características, a maior produção será alcançada com a aplicação de doses superiores a 200 $\mathrm{mg} \mathrm{dm}{ }^{-3}$. Para a altura, o efeito foi curvilíneo, descrito por modelo hiperbólico. Já o PMSR não sofreu efeito, em razão da aplicação de $\mathrm{N}$ nesse substrato.

No solo PVA houve efeito linear positivo apenas quanto às características diâmetro e PMSPA.

As menores médias das diversas características estudadas foram verificadas no substrato LVD. Efeito significativo só foi observado no PMSPA, sendo de ordem quadrática.

Efeitos no crescimento de plantas em virtude da aplicação de N são esperados, em razão das diversas funções que o nutriente exerce dentro delas. Assim como neste estudo, Crestana et al. (1995) também verificaram efeito positivo da adubação nitrogenada sobre a característica diâmetro quando utilizou suprimento de uréia, cultivando diversas espécies florestais. Marques et al. (2006), estudando diferentes fontes de $\mathrm{N}$ na produção de mudas de angico-vermelho, verificaram que a dose de $180 \mathrm{mg} \cdot \mathrm{dm}^{-3}$ foi a que proporcionou melhor desenvolvimento e crescimento para a maioria das características estudadas.

Os poucos efeitos da aplicação de $\mathrm{N}$ observados no PVAe no LVD também já foram, entretanto, observados em diversos trabalhos, como o de Oliveira et al. (1998), no crescimento de angelim-pedra (Dinizia excelsa DUCKE); e em todas as características avaliadas das mudas de guatambu (Aspidosperma olivaceum) e copaíba (Copaifera langsdorffii) por Paron et al. (1996).

Quadro 3 - Estimativas dos contrastes médios entre o tratamento sem adição de sais e os demais tratamentos, em que se adicionaram macronutrientes

Table 3 - Estimates of the mean contrasts between the treatment without addition of salts and the other treatments, with added macronutrients

\begin{tabular}{lccccc}
\hline \multicolumn{5}{c}{ Estimativas do contraste } \\
\hline SOLO & DIAM & ALTURA & PMSPA & PMSR & PMST \\
LVD & $40,3 * * *$ & $248,09 * * *$ & $41,72 * * *$ & $56,50 * * *$ & $96,73 * * *$ \\
PVA & $69,49 * * *$ & $596,18 * * *$ & $117,64 * * *$ & $170,72 * * *$ \\
LVA & $72,63 * * *$ & $812,83 * * *$ & $132,69 * * *$ & $155,83 * * *$ & $288,36 * * *$ \\
\hline
\end{tabular}

$* * *$ Significativo a $1 \%$ de probabilidade.

R. Árvore, Viçosa-MG, v.32, n.6, p.1029-1040, 2008 
Crescimento de mudas de angico-vermelho ...

Quadro 4 - Médias do angico-vermelho em razão da aplicação de N, P, K, Ca, Mg e S das seguintes características: altura (ALT), diâmetro (DIAM), peso de matéria seca da parte aérea (PMSPA), peso de matéria seca de raiz (PMSR) e peso de matéria seca total (PMST)

Table 4-Means for angico vermelho, after application of $N, P, K, C a, M g$ and S for the following characteristics: height (ALT), diameter (DIAM), weight of dry matter of the aerial part (PMSPA), root dry matter weight (PMSR), Total dry matter weight (PMST)

\begin{tabular}{|c|c|c|c|c|c|c|}
\hline SOLO & $\mathrm{N}\left(\mathrm{mg} \mathrm{dm}^{-3}\right)$ & DIAM $(\mathrm{mm})$ & $\operatorname{ALT}(\mathrm{cm})$ & $\operatorname{PMSPA}(\mathrm{g})$ & $\operatorname{PMSR}(\mathrm{g})$ & $\operatorname{PMST}(\mathrm{g})$ \\
\hline \multirow[t]{4}{*}{ LVD } & 50 & 3,61 & 22,53 & 1,49 & 2,96 & 4,45 \\
\hline & 100 & 3,81 & 28,00 & 2,53 & 3,07 & 5,60 \\
\hline & 150 & 3,99 & 33,63 & 3,00 & 3,60 & 6,61 \\
\hline & 200 & 4,10 & 30,88 & 1,93 & 3,57 & 5,50 \\
\hline \multirow[t]{4}{*}{ PVA } & 50 & 5,91 & 39,45 & 4,70 & 9,08 & 13,79 \\
\hline & 100 & 6,23 & 41,48 & 6,15 & 9,92 & 16,07 \\
\hline & 150 & 7,33 & 55,55 & 9,42 & 9,45 & 18,86 \\
\hline & 200 & 7,35 & 46,53 & 8,35 & 8,82 & 17,17 \\
\hline \multirow[t]{4}{*}{ LVA } & 50 & 5,66 & 39,60 & 3,93 & 8,59 & 12,52 \\
\hline & 100 & 7,18 & 64,53 & 8,33 & 10,20 & 18,54 \\
\hline & 150 & 7,10 & 53,75 & 9,31 & 8,38 & 17,68 \\
\hline & 200 & 7,38 & 70,38 & 12,72 & 11,51 & 24,22 \\
\hline$\overline{\text { SOLO }}$ & $\mathrm{P}\left(\mathrm{mg} \mathrm{dm}^{-3}\right)$ & DIAM $(\mathrm{mm})$ & ALT $(\mathrm{cm})$ & PMSPA (g) & PMSR (g) & $\overline{\operatorname{PMST}(\mathrm{g})}$ \\
\hline \multirow[t]{4}{*}{ LVD } & 150 & 2,70 & 17,35 & 0,63 & 2,05 & 2,68 \\
\hline & 300 & 3,81 & 28,00 & 2,53 & 3,07 & 5,60 \\
\hline & 450 & 3,94 & 27,69 & 2,46 & 3,30 & 5,76 \\
\hline & 600 & 4,42 & 28,75 & 2,91 & 3,21 & 6,12 \\
\hline \multirow[t]{4}{*}{ PVA } & 150 & 6,49 & 36,53 & 5,34 & 10,07 & 15,41 \\
\hline & 300 & 6,23 & 41,48 & 6,15 & 9,92 & 16,07 \\
\hline & 450 & 6,56 & 49,35 & 6,85 & 7,28 & 14,13 \\
\hline & 600 & 7,01 & 46,75 & 7,14 & 6,77 & 13,91 \\
\hline \multirow[t]{4}{*}{ LVA } & 150 & 5,03 & 29,98 & 3,69 & 6,54 & 10,23 \\
\hline & 300 & 7,18 & 64,53 & 8,33 & 10,20 & 18,54 \\
\hline & 450 & 6,68 & 64,30 & 8,88 & 6,98 & 15,85 \\
\hline & 600 & 6,63 & 55,48 & 6,97 & 7,29 & 14,27 \\
\hline SOLO & $\mathrm{K}\left(\mathrm{mg} \mathrm{dm}^{-3}\right)$ & DIAM $(\mathrm{mm})$ & $\operatorname{ALT}(\mathrm{cm})$ & PMSPA $(\mathrm{g})$ & PMSR (g) & $\operatorname{PMST}(\mathrm{g})$ \\
\hline \multirow[t]{4}{*}{ LVD } & 50 & 4,62 & 28,98 & 3,52 & 3,90 & 7,42 \\
\hline & 100 & 3,81 & 28,00 & 2,53 & 3,07 & 5,60 \\
\hline & 150 & 3,25 & 24,55 & 1,97 & 2,75 & 4,72 \\
\hline & 200 & 4,26 & 29,40 & 2,73 & 3,28 & 6,01 \\
\hline \multirow[t]{4}{*}{ PVA } & 50 & 6,20 & 44,15 & 4,95 & 11,39 & 16,34 \\
\hline & 100 & 6,23 & 41,48 & 6,15 & 9,92 & 16,07 \\
\hline & 150 & 5,80 & 38,28 & 5,43 & 8,96 & 14,39 \\
\hline & 200 & 6,65 & 48,75 & 7,67 & 11,99 & 19,65 \\
\hline \multirow[t]{4}{*}{ LVA } & 50 & 6,79 & 61,60 & 8,48 & 9,48 & 17,96 \\
\hline & 100 & 7,18 & 64,53 & 8,33 & 10,20 & 18,54 \\
\hline & 150 & 6,29 & 63,06 & 6,27 & 8,68 & 14,95 \\
\hline & 200 & 6,75 & 63,06 & 7,69 & 9,45 & 17,15 \\
\hline$\overline{\mathrm{SOLO}}$ & $\mathrm{Ca}\left(\mathrm{cmol}_{\mathrm{c}} \mathrm{dm}^{-3}\right)$ & DIAM $(\mathrm{mm})$ & ALT $(\mathrm{cm})$ & PMSPA (g) & PMSR (g) & $\overline{P M S T}(\mathrm{~g})$ \\
\hline \multirow[t]{4}{*}{$\overline{\mathrm{LVD}}$} & 0,8 & 4,06 & 24,38 & 2,52 & 4,07 & 5,57 \\
\hline & 1 & 3,81 & 28,00 & 2,53 & 3,07 & 5,60 \\
\hline & 1,2 & 4,74 & 32,53 & 3,27 & 3,08 & 8,28 \\
\hline & 1,4 & 3,42 & 19,20 & 1,51 & 3,18 & 4,68 \\
\hline \multirow[t]{4}{*}{ PVA } & 0,8 & 6,32 & 41,28 & 6,52 & 9,53 & 16,05 \\
\hline & 1 & 6,23 & 41,48 & 6,15 & 9,92 & 16,07 \\
\hline & 1,2 & 7,03 & 49,18 & 6,92 & 10,30 & 17,22 \\
\hline & 1,4 & 5,15 & 39,60 & 4,64 & 7,65 & 12,28 \\
\hline
\end{tabular}


Quadro 4-Cont.

Table 4-Cont.

\begin{tabular}{lcccccc}
\hline SOLO & $\mathrm{Ca}\left(\mathrm{cmol}_{\mathrm{c}} \mathrm{dm}^{-3}\right)$ & DIAM $(\mathrm{mm})$ & ALT $(\mathrm{cm})$ & PMSPA $(\mathrm{g})$ & PMSR $(\mathrm{g})$ & PMST $(\mathrm{g})$ \\
\hline LVA & 0,8 & 6,16 & 43,60 & 6,63 & 7,57 & 16,01 \\
& 1 & 7,18 & 64,53 & 8,33 & 10,20 & 18,54 \\
& 1,2 & 5,84 & 52,05 & 6,04 & 8,08 & 14,12 \\
& 1,4 & 6,98 & 58,18 & 8,52 & 9,08 & 17,60 \\
\hline SOLO & $\mathrm{Mg}\left(\mathrm{cmol}_{\mathrm{c}} \mathrm{dm}^{-3}\right)$ & DIAM $(\mathrm{mm})$ & ALT $(\mathrm{cm})$ & PMSPA $(\mathrm{g})$ & PMSR $(\mathrm{g})$ & PMST $(\mathrm{g})$ \\
\hline LVD & 0,2 & 4,38 & 27,43 & 2,36 & 3,57 & 5,94 \\
& 0,4 & 3,81 & 28,00 & 2,53 & 3,07 & 5,60 \\
& 0,6 & 4,39 & 32,38 & 3,23 & 4,20 & 7,43 \\
PVA & 0,8 & 3,50 & 22,13 & 1,63 & 2,10 & 3,73 \\
& 0,2 & 6,60 & 41,13 & 6,76 & 8,95 & 15,71 \\
& 0,4 & 6,23 & 41,48 & 6,15 & 9,92 & 16,07 \\
& 0,6 & 6,27 & 43,35 & 6,19 & 10,26 & 16,45 \\
LVA & 0,8 & 6,59 & 52,73 & 7,09 & 9,97 & 17,06 \\
& 0,2 & 6,68 & 63,70 & 6,39 & 7,57 & 13,95 \\
& 0,4 & 7,18 & 64,53 & 8,33 & 10,20 & 18,54 \\
& 0,6 & 7,15 & 62,58 & 8,02 & 10,03 & 18,06 \\
SOLO & 0,8 & 6,82 & 56,50 & 8,22 & 11,19 & 19,41 \\
\hline LVD & $\mathrm{S}\left(\mathrm{mg} \mathrm{dm}^{-3}\right)$ & DIAM $(\mathrm{mm})$ & $\mathrm{ALT}(\mathrm{cm})$ & PMSPA $(\mathrm{g})$ & PMSR $(\mathrm{g})$ & PMST $(\mathrm{g})$ \\
& 20 & 4,03 & 18,88 & 1,21 & 3,32 & 4,54 \\
& 40 & 3,81 & 28,00 & 2,53 & 3,07 & 5,60 \\
& 60 & 3,78 & 23,45 & 1,47 & 4,36 & 4,74 \\
PVA & 80 & 5,08 & 36,78 & 4,04 & 5,46 & 9,50 \\
& 20 & 6,53 & 41,90 & 7,05 & 10,96 & 18,01 \\
& 40 & 6,23 & 41,48 & 6,15 & 9,92 & 16,07 \\
& 60 & 6,31 & 46,40 & 7,62 & 10,79 & 18,41 \\
LVA & 80 & 6,78 & 39,40 & 6,32 & 11,63 & 17,95 \\
& 20 & 6,99 & 51,28 & 7,26 & 11,17 & 18,43 \\
& 40 & 7,18 & 64,53 & 8,33 & 10,20 & 18,54 \\
& 60 & 4,91 & 45,18 & 4,65 & 6,46 & 11,10 \\
& 80 & 7,46 & 47,28 & 7,76 & 9,23 & 16,99 \\
\hline
\end{tabular}

Quadro 5 - Resumo das estimativas geradas do efeito do N em mudas de angico-vermelho aos 120 dias após a semeadura, considerando a altura, diâmetro e peso de matéria seca da raiz, parte aérea e total

Table 5 - Summary of the estimates generated from the effect of $\mathrm{N}$ on angico vermelho seedlings at 120 days after sowing, considering height, diameter, and dry matter weight of the root, aerial and total part

\begin{tabular}{|c|c|c|c|c|}
\hline Característica & Solo & Equação & $\mathrm{R}^{2}$ & Dose recomendada $\left(\mathrm{mg} / \mathrm{dm}^{3}\right)$ \\
\hline \multirow[t]{3}{*}{$\overline{\text { DIAM }(\mathrm{mm})}$} & LVD & $\hat{\mathrm{Y}}=\overline{\mathrm{Y}}=3,88$ & & 50 \\
\hline & PVA & $\hat{\mathrm{Y}}=5,35+0,0108 * * \mathrm{X}$ & 0,88 & 200 \\
\hline & LVA & $\hat{\hat{Y}}=5,55+0,0102 * * X$ & 0,69 & 200 \\
\hline \multirow[t]{3}{*}{ ALTURA (cm) } & LVD & $\hat{\mathrm{Y}}=\overline{\bar{Y}}=28,76$ & & 50 \\
\hline & PVA & $\hat{\hat{Y}}=\overline{\bar{Y}}=45,75$ & & 50 \\
\hline & LVA & $\hat{\hat{Y}}=74,77-1700(1 / * * X)$ & 0,48 & 200 \\
\hline \multirow[t]{3}{*}{$\operatorname{PMSPA}(\mathrm{g})$} & LVD & $\hat{\hat{Y}}=-0,84+0,0562 * X-0,0002 * X^{2}$ & 0,96 & 102 \\
\hline & PVA & $\hat{\hat{Y}}=3,60+0,284 * * * X$ & 0,74 & 200 \\
\hline & LVA & $\hat{\hat{Y}}=1,74+0,0546 * * * X$ & 0,95 & 200 \\
\hline \multirow[t]{3}{*}{$\operatorname{PMSR}(\mathrm{g})$} & LVD & $\hat{\mathrm{Y}}=\overline{\mathrm{Y}}=3,30$ & & 50 \\
\hline & PVA & $\hat{\mathrm{Y}}=\underline{\mathrm{Y}}=9,32$ & & 50 \\
\hline & LVA & $\hat{\hat{Y}}=\overline{\mathrm{Y}}=9,67$ & & 50 \\
\hline \multirow[t]{3}{*}{$\operatorname{PMST}(\mathrm{g})$} & LVD & $\hat{\mathrm{Y}}=\hat{\mathrm{Y}}=5,54$ & & 50 \\
\hline & PVA & $\hat{\mathrm{Y}}=\overline{\mathrm{Y}}=16,47$ & & 50 \\
\hline & LVA & $\hat{\hat{Y}}=9,68+0,0685 * * * X$ & 0,85 & 200 \\
\hline
\end{tabular}

\footnotetext{
*** Significativo a $1 \%$ de probabilidade; ** Significativo a $5 \%$ de probabilidade; e *Significativo a $10 \%$ de probabilidade.
}

R. Árvore, Viçosa-MG, v.32, n.6, p.1029-1040, 2008 


\subsection{Efeito do Fósforo}

$\mathrm{O} P$ foi o nutriente que mais proporcionou efeitos significativos no crescimento das plantas, implicando aumento dos valores observados de todas as características avaliadas (Quadro 4).

Pelo Quadro 6, observa-se no substrato LVD que o P proporcionou efeito linear positivo para o diâmetro, a altura e o PMSPA. Em contrapartida, no PVA ocorreu efeito linear negativo na característica PMSR. Embora a aplicação de P nesse substrato (PVA) tenha provocado poucos efeitos significativos, as médias observadas são maiores do que as médias observadas no substrato LVA, nas doses menores; isso indica que, na ausência de adubações, ele seria melhor do que o LVA.

No LVA houve efeito significativo de todas as características avaliadas, sendo o efeito de ordem raiz quadrada, em que o aumento de doses favoreceu o aumento dos valores das características até um ponto de máximo, em doses que variaram de 206 a 279 mg $\mathrm{dm}^{-3}$, quando, então, as médias diminuíam.

A exemplo deste estudo, Ceconi et al. (2006) verificaram que o melhor crescimento das mudas de açoita-cavalo (Luehea divaricata) ocorreu com a dose de $360 \mathrm{mg} \mathrm{dm}^{-3}$ de fósforo. Outro efeito da aplicação de $\mathrm{P}$ com resposta positiva foram verificados por Nicoloso et al. (2001) e Missio et al. (2004) em mudas de grápia.

\subsection{Efeito do Potássio}

Em razão de pouca variação nos valores médios observados (Quadro 4), o K foi o nutriente que provocou menores efeitos significativos nas diversas características estudadas (Quadro 7), os quais foram observados no LVD e no LVA e ausentes no PVA.

Tais resultados indicam que a necessidade da planta por esse nutriente pode ter sido suprida pelos teores que estavam originalmente no solo (PVA = $14 \mathrm{mg} \mathrm{dm}^{-3} ; \mathrm{LVA}=32 \mathrm{mg} \mathrm{dm}^{-3} ; \mathrm{LVD}=49 \mathrm{mg} \mathrm{dm}^{-3}$ ) e, ou, a espécie possui baixa exigência nutricional por esse elemento.

No LVA, o efeito foi linear e negativo para o PMSPA, indicando que em doses maiores de K o desenvolvimento da parte aérea foi prejudicado. Nessa mesma característica, Venturin et al. (1999) também verificaram menor produção em mudas de angico-amarelo (Peltophorum dubium), em razão da aplicação de K.

Quadro 6 - Resumo das estimativas geradas para efeito do P em mudas de angico-vermelho aos 120 dias após a semeadura, considerando a altura, diâmetro e peso de matéria seca da raiz, parte aérea e total

Table 6 - Summary of the estimates generated for $P$ effect on angico vermelho seedlings at 120 days after sowing, considering height, diameter, and dry matter weight of the root, aerial and total part

\begin{tabular}{|c|c|c|c|c|}
\hline Característica & Solo & Equação & $\mathrm{R}^{2}$ & Dose recomendada $\left(\mathrm{mg} / \mathrm{dm}^{3}\right)$ \\
\hline \multirow[t]{3}{*}{$\overline{D I A M}(\mathrm{~mm})$} & LVD & $\hat{Y}=2,3925+0,0035 X$ & 0,88 & 600 \\
\hline & PVA & $\hat{\mathrm{Y}}=\overline{\mathrm{Y}}=6,57$ & & 150 \\
\hline & LVA & $\hat{Y}=-6,03924+1,3090^{* *} X^{0,5}-0,0325856^{* *} X$ & 0,45 & 238,11 \\
\hline \multirow[t]{3}{*}{ ALTURA $(\mathrm{cm})$} & LVD & $\hat{\mathrm{Y}}=2,3925+0,0035 * * \mathrm{X}$ & 0,88 & 600 \\
\hline & PVA & $\hat{\mathrm{Y}}=\overline{\mathrm{Y}}=43,53$ & & 150 \\
\hline & LVA & $\hat{Y}=-178,926+24,6442 * * X^{0,5}-0,616762 * * X$ & 0,63 & 278,45 \\
\hline \multirow[t]{3}{*}{ PMSPA (g) } & LVD & $\hat{Y}=0,4388+0,0045 * * X$ & 0,73 & 600 \\
\hline & PVA & $\hat{Y}=\bar{Y}=6,37$ & & 150 \\
\hline & LVA & $\hat{Y}=-27,1433+3,6388 * X^{0,5}-0,0916495 * X$ & 0,67 & 279,35 \\
\hline \multirow[t]{3}{*}{$\operatorname{PMSR}(\mathrm{g})$} & LVD & $\hat{\mathrm{Y}}=\overline{\mathrm{Y}}=2,91$ & & 150 \\
\hline & PVA & $\hat{Y}=11,646-0,084 * X$ & 0,88 & 600 \\
\hline & LVA & $\hat{Y}=-11,008+2,19437 * X^{0,5}-0,0600905^{*} X$ & 0,20 & 206,83 \\
\hline \multirow[t]{3}{*}{$\operatorname{PMST}(\mathrm{g})$} & LVD & $\hat{Y}=\bar{Y}=5,04$ & & 150 \\
\hline & PVA & $\hat{\mathrm{Y}}=\overline{\mathrm{Y}}=14,88$ & & 150 \\
\hline & LVA & $\hat{Y}=-38,1520+5,83320 * * X^{0,5}-0,151740^{* *} X$ & 0,53 & 249,27 \\
\hline
\end{tabular}

*** Significativo a $1 \%$ de probabilidade; $* *$ Significativo a $5 \%$ de probabilidade; e $*$ Significativo a $10 \%$ de probabilidade. 
Quadro 7 - Resumo das estimativas geradas para efeito do K em mudas de angico-vermelho aos 120 dias após a semeadura, considerando a altura, diâmetro e peso de matéria seca da raiz, peso da matéria seca da parte aérea e peso da matéria seca total

Table 7 - Summary of the estimates generated for the effect of $K$ on angico vermelho seedlings at 120 days after sowing, considering height, diameter, and dry matter weight of the root, aerial and total part

\begin{tabular}{|c|c|c|c|c|}
\hline Característica & Solo & Equação & $\mathrm{R}^{2}$ & Dose recomendada $\left(\mathrm{mg} / \mathrm{dm}^{3}\right)$ \\
\hline \multirow[t]{3}{*}{ DIAM (mm) } & LVD & $\hat{Y}=+6,6644-0,0486 * X+0,0002 * X^{2}$ & 0,92 & 121,5 \\
\hline & PVA & $\hat{\mathrm{Y}}=\overline{\mathrm{Y}}=6,22$ & & 50 \\
\hline & LVA & $\hat{\mathrm{Y}}=\overline{\mathrm{Y}}=6,75$ & & 50 \\
\hline \multirow[t]{3}{*}{$\operatorname{ALTURA}(\mathrm{cm})$} & LVD & $\hat{Y}=\bar{Y}=27,73$ & & 50 \\
\hline & PVA & $\hat{\mathrm{Y}}=\overline{\mathrm{Y}}=43,16$ & & 50 \\
\hline & LVA & $\hat{\mathrm{Y}}=\overline{\mathrm{Y}}=63,06$ & & 50 \\
\hline \multirow[t]{3}{*}{ PMSPA (g) } & LVD & $\hat{\mathrm{Y}}=\overline{\mathrm{Y}}=2,39$ & & 50 \\
\hline & PVA & $\hat{\mathrm{Y}}=\overline{\mathrm{Y}}=6,05$ & & 50 \\
\hline & LVA & $\hat{\mathrm{Y}}=8,8017-0,008 * * \mathrm{X}(-)$ & 0,32 & 50 \\
\hline \multirow[t]{3}{*}{ PMSR (g) } & LVD & $\hat{\mathrm{Y}}=\overline{\mathrm{Y}}=3,25$ & & 50 \\
\hline & PVA & $\hat{\mathrm{Y}}=\overline{\mathrm{Y}}=10,56$ & & 50 \\
\hline & LVA & $\hat{\mathrm{Y}}=\overline{\mathrm{Y}}=9,45$ & & 50 \\
\hline \multirow[t]{3}{*}{ PMST (g) } & LVD & $\hat{\mathrm{Y}}=\overline{\mathrm{Y}}=5,94$ & & 50 \\
\hline & PVA & $\hat{\mathrm{Y}}=\overline{\mathrm{Y}}=16,61$ & & 50 \\
\hline & LVA & $\hat{\mathrm{Y}}=\overline{\mathrm{Y}}=17,15$ & & 50 \\
\hline
\end{tabular}

$* * *$ Significativo a $1 \%$ de probabilidade; $* *$ Significativo a $5 \%$ de probabilidade; e $*$ Significativo a $10 \%$ de probabilidade.

O fato de no PVA não terem sido detectados efeitos significativos, mesmo com os teores de $14 \mathrm{mg}$ de K $\mathrm{dm}^{-3}$ encontrados originalmente no solo, corrobora os dados de Novais et al. (1979), que notaram ausência de resposta à aplicação de potássio em mudas de Eucalyptus grandis, ainda que o nível de K no solo estivesse em $4 \mathrm{mg} \mathrm{dm}^{-3}$ e também com os de Balieiro et al. (2001) em mudas de Acacia holosericea e Acacia auriculiformis, que verificaram ausência de efeitos sob aplicação de K com relação às características diâmetro do caule, altura e matéria seca da parte aérea.

\subsection{Efeito do Cálcio}

A espécie apresentou baixa resposta à aplicação de Ca, nos substratos estudados, sendo as médias apresentadas no Quadro 4. Efeitos significativos só foram observados nas características diâmetro no LVA e altura no LVD, ambos com efeito quadrático (Quadro 8).

No caso do PVA, a ausência de efeitos nas diversas características pode estar relacionada ao alto teor de Ca que esse solo apresentava inicialmente, cerca de $1,60 \mathrm{cmol}_{\mathrm{c}} \mathrm{dm}^{-3}$, valor esse superior à maior dose testada.
Em estudos de outros autores, essa falta de resposta em razão da aplicação de Ca também foi verificada, como em mudas de jatobá (Hymenaea courbaril), estudadas por Duboc et al. (1996).

Entretanto, resposta à aplicação de Ca já foi observada em outras espécies, como em mudas de Eucalyptus citriodora, que apresentaram menor crescimento em altura, em razão da omissão de Ca (MAFFEIS et al., 2000).

\subsection{Efeito do Magnésio}

O crescimento das plantas sob condições diferenciadas de $\mathrm{Mg}$ só se mostrou diferente para o PMSR e PMST no LVA (Quadro 9), sendo as médias apresentadas no Quadro 4.

O efeito observado no LVA nas características mencionadas foi linear e positivo. Portanto, maior crescimento e desenvolvimento são esperados em doses superiores a $0,8 \mathrm{cmol}_{\mathrm{c}}$ de $\mathrm{Mg} \mathrm{dm}^{-3}$ de solo. Devendo-se, então, no processo de produção de mudas, em solos com essa característica, utilizar doses superiores a esse valor. 
Quadro 8 - Resumo das estimativas geradas para efeito do Ca em mudas de angico-vermelho aos 120 dias após a semeadura, considerando a altura, diâmetro e peso de matéria seca da raiz, parte aérea e total

Table 8 -Summary of the estimates generated for the effect of Ca on angico vermelho seedlings at 120 days after sowing, considering height, diameter, dry matter weight of the root, aerial and total part

\begin{tabular}{|c|c|c|c|c|}
\hline Característica & Solo & Equação & $\mathrm{R}^{2}$ & Dose recomendada $\left(\mathrm{cmol}_{\mathrm{c}} / \mathrm{dm}^{3}\right)$ \\
\hline \multirow[t]{3}{*}{ DIAM (mm) } & LVD & $\hat{\mathrm{Y}}=\overline{\mathrm{Y}}=4,00$ & & 0,8 \\
\hline & PVA & $\hat{\mathrm{Y}}=\overline{\mathrm{Y}}=6,54$ & & 0,8 \\
\hline & LVA & $\hat{Y}=-5,2656+23,166 * X-11,141 * X^{2}$ & 0,64 & 0,8 \\
\hline \multirow[t]{3}{*}{ ALTURA $(\mathrm{cm})$} & LVD & $\hat{Y}=-90,813+227,56 * X-105,94 * X^{2}$ & 0,81 & 0,9 \\
\hline & PVA & $\hat{\mathrm{Y}}=\overline{\mathrm{Y}}=42,88$ & & 0,8 \\
\hline & LVA & $\hat{\mathrm{Y}}=\overline{\mathrm{Y}}=54,59$ & & 0,8 \\
\hline \multirow[t]{3}{*}{ PMSPA (g) } & LVD & $\hat{\mathrm{Y}}=\overline{\mathrm{Y}}=2,45$ & & 0,8 \\
\hline & PVA & $\hat{\mathrm{Y}}=\overline{\mathrm{Y}}=6,05$ & & 0,8 \\
\hline & LVA & $\hat{\mathrm{Y}}=\overline{\mathrm{Y}}=7,34$ & & 0,8 \\
\hline \multirow[t]{3}{*}{$\operatorname{PMSR}(\mathrm{g})$} & LVD & $\hat{\mathrm{Y}}=\overline{\mathrm{Y}}=3,35$ & & 0,8 \\
\hline & PVA & $\hat{\mathrm{Y}}=\overline{\mathrm{Y}}=9,35$ & & 0,8 \\
\hline & LVA & $\hat{\mathrm{Y}}=\overline{\mathrm{Y}}=8,73$ & & 0,8 \\
\hline \multirow[t]{3}{*}{ PMST (g) } & LVD & $\hat{Y}=\bar{Y}=6,03$ & & 0,8 \\
\hline & PVA & $\hat{Y}=\bar{Y}=15,40$ & & 0,8 \\
\hline & LVA & $\hat{Y}=\bar{Y}=16,53$ & & 0,8 \\
\hline
\end{tabular}

*** Significativo a $1 \%$ de probabilidade; ** Significativo a $5 \%$ de probabilidade; e *Significativo a $10 \%$ de probabilidade.

Quadro 9-Resumo das estimativas geradas para efeito do Mg em mudas de angico-vermelho aos 120 dias após a semeadura, considerando a altura, diâmetro e peso de matéria seca da raiz, parte aérea e total

Table 9 - Summary of the estimates generated for Mg effect on angico vermelho seedlings at 120 days after sowing, considering height, diameter, dry matter weight of the root, aerial and total part

\begin{tabular}{|c|c|c|c|c|}
\hline Característica & Solo & Equação & $\mathrm{R}^{2}$ & Dose recomendada $\left(\mathrm{cmol}_{\mathrm{c}} / \mathrm{dm}^{3}\right)$ \\
\hline \multirow[t]{3}{*}{$\overline{\text { DIAM (mm) }}$} & LVD & $\hat{\mathrm{Y}}=\mathrm{Y}=4,02$ & & 0,2 \\
\hline & PVA & $\hat{\mathrm{Y}}=\overline{\mathrm{Y}}=6,42$ & & 0,2 \\
\hline & LVA & $\hat{\mathrm{Y}}=\overline{\mathrm{Y}}=6,69$ & & 0,2 \\
\hline \multirow[t]{3}{*}{$\operatorname{ALTURA}(\mathrm{cm})$} & LVD & $\hat{\mathrm{Y}}=\overline{\mathrm{Y}}=27,48$ & & 0,2 \\
\hline & PVA & $\hat{\mathrm{Y}}=\overline{\mathrm{Y}}=44,67$ & & 0,2 \\
\hline & LVA & $\hat{\mathrm{Y}}=\overline{\mathrm{Y}}=61,83$ & & 0,2 \\
\hline \multirow[t]{3}{*}{ PMSPA (g) } & LVD & $\hat{\mathrm{Y}}=\overline{\mathrm{Y}}=2,44$ & & 0,2 \\
\hline & PVA & $\hat{\mathrm{Y}}=\overline{\mathrm{Y}}=6,55$ & & 0,2 \\
\hline & LVA & $\hat{\mathrm{Y}}=\overline{\mathrm{Y}}=7,74$ & & 0,2 \\
\hline \multirow[t]{3}{*}{$\operatorname{PMSR}(\mathrm{g})$} & LVD & $\hat{\mathrm{Y}}=\overline{\mathrm{Y}}=3,24$ & & 0,2 \\
\hline & PVA & $\hat{\mathrm{Y}}=\overline{\mathrm{Y}}=9,77$ & & 0,2 \\
\hline & LVA & $\hat{Y}=7,075+5,345 * X$ & 0,80 & 0,8 \\
\hline \multirow[t]{3}{*}{ PMST (g) } & LVD & $\hat{\mathrm{Y}}=\overline{\mathrm{Y}}=5,67$ & & 0,2 \\
\hline & PVA & $\hat{\mathrm{Y}}=\overline{\mathrm{Y}}=16,32$ & & 0,2 \\
\hline & LVA & $\hat{\mathrm{Y}}=13,515+7,9462 * X$ & 0,72 & 0,8 \\
\hline
\end{tabular}

*** Significativo a $1 \%$ de probabilidade; $* *$ Significativo a $5 \%$ de probabilidade; e *Significativo a $10 \%$ de probabilidade. 
O fato de poucos efeitos significativos terem sido observados indicam que a espécie em questão tem baixa exigência pelo $\mathrm{Mg}$. Tal comportamento também já foi verificado nas espécies de jacaré (Piptadenia gonoacantha) e canafístula (Senna multijuga) estudadas por Renó et al. (1997).

\subsection{Efeito do Enxofre}

Após a análise dos dados, cujas médias se encontram no Quadro 4, verificou-se que não houve diferenças significativas sobre o diâmetro das plantas em nenhuma classe de solo. Nas demais características avaliadas, apenas as mudas cultivadas no solo LVD apresentaram crescimento diferenciado de forma significativa. Nessa situação, as mudas tiveram as médias de crescimento das diversas características aumentadas de forma linear (Quadro 10).

No LVA e no PVA, o suprimento de S não implicou efeitos significativos sobre o crescimento das mudas de angico, nas diversas características estudadas. Tal fato já foi observado em mudas de táxi-branco (Sclerolobium paniculatum) cultivadas por Dias et al. (1992). Similarmente, em outro estudo, plantas de jatobá (Hymenaea courbaril) cultivadas em um Latossolo Vermelho-Amarelo com baixa disponibilidade de nutrientes os valores de altura, diâmetro, matéria seca de parte aérea e raiz não diferiram estatisticamente dos obtidos no tratamento completo (DUBOC et al., 1996).

No solo LVD, cujos efeitos foram lineares e positivos em todas as características estudadas, exceto o diâmetro, a quantidade ideal de $S$ a ser aplicada, para garantir maior produtividade está em valores superiores a $80 \mathrm{mg} / \mathrm{dm}^{3}$. Esses resultados estão de acordo com os de Maffeis et al. (2000) em mudas de Eucalyptus citriodora, em que a omissão do $S$ fez que estas tivessem menor crescimento em altura e diâmetro, bem como menor biomassa de folhas (g/planta), comparativamente ao obtido nas mudas produzidas em solução completa. Da mesma forma, o nutriente foi altamente limitante para o crescimento de mudas de cedro (Cedrela fissilis), jacaré (Piptadenia gonoacantha), pau-ferro (Caesalpinea ferrea) e canafístula (Senna multijuga) em um Latossolo Vermelho-Amarelo de fertilidade natural muito baixa, conforme foi verificado por Renó et al. (1997).

Quadro 10 - Resumo das estimativas geradas para efeito do S em mudas de angico-vermelho aos 120 dias após a semeadura, considerando a altura, diâmetro e peso de matéria seca da raiz, parte aérea e total

Table 10 - Summary of the estimates generated for S effect on angico vermelho seedlings at 120 days after sowing, considering height, diameter, dry matter weight of the root, aerial and total part

\begin{tabular}{|c|c|c|c|c|}
\hline Característica & Solo & Equação & $\mathrm{R}^{2}$ & Dose recomendada $\left(\mathrm{mg} / \mathrm{dm}^{3}\right)$ \\
\hline \multirow[t]{3}{*}{ DIAM (mm) } & LVD & $\hat{\mathrm{Y}}=\overline{\mathrm{Y}}=4,17$ & & 20 \\
\hline & PVA & $\hat{\mathrm{Y}}=\overline{\mathrm{Y}}=6,46$ & & 20 \\
\hline & LVA & $\hat{Y}=\bar{Y}=6,63$ & & 20 \\
\hline \multirow[t]{3}{*}{ ALTURA $(\mathrm{cm})$} & LVD & $\hat{Y}=14,488+0,2458 * * * X$ & 0,69 & 80 \\
\hline & PVA & $\hat{Y}=\bar{Y}=42,29$ & & 20 \\
\hline & LVA & $\hat{\mathrm{Y}}=\overline{\mathrm{Y}}=52,06$ & & 20 \\
\hline \multirow[t]{3}{*}{ PMSPA (g) } & LVD & $\hat{Y}=0,4563+0,0371 * * * X$ & 0,56 & 80 \\
\hline & PVA & $\hat{\mathrm{Y}}=\overline{\mathrm{Y}}=6,79$ & & 20 \\
\hline & LVA & $\hat{\mathrm{Y}}=\overline{\mathrm{Y}}=7,00$ & & 20 \\
\hline \multirow[t]{3}{*}{$\operatorname{PMSR}(\mathrm{g})$} & LVD & $\hat{\mathrm{Y}}=2,13+0,0385 * \mathrm{X}$ & 0,83 & 80 \\
\hline & PVA & $\hat{\mathrm{Y}}=\overline{\mathrm{Y}}=10,82$ & & 20 \\
\hline & LVA & $\hat{\mathrm{Y}}=\overline{\mathrm{Y}}=9,26$ & & 20 \\
\hline \multirow[t]{3}{*}{$\operatorname{PMST}(\mathrm{g})$} & LVD & $\hat{Y}=2,5863+0,0701 * * X$ & 0,61 & 80 \\
\hline & PVA & $\hat{\mathrm{Y}}=\overline{\mathrm{Y}}=17,61$ & & 20 \\
\hline & LVA & $\hat{\mathrm{Y}}=\overline{\mathrm{Y}}=16,26$ & & 20 \\
\hline
\end{tabular}

$* * *$ Significativo a $1 \%$ de probabilidade; ** Significativo a $5 \%$ de probabilidade; e $*$ Significativo a $10 \%$ de probabilidade.

R. Árvore, Viçosa-MG, v.32, n.6, p.1029-1040, 2008 


\section{CONCLUSÕES E RECOMENDAÇÕES}

A aplicação de macronutrientes refletiu maior crescimento e desenvolvimento das mudas de angicovermelho.

O melhor solo para produção das mudas entre os três estudados é o Latossolo Vermelho-amarelo álico.

Fundamentando nos dados de matéria seca total, que foi considerada como a característica que melhor reflete a produção, as doses de macronutrientes recomendadas para produção de mudas são: $\mathrm{N}$ (50 a $\left.200 \mathrm{mg} \mathrm{dm}^{-3}\right)$; P (150 a $\left.250 \mathrm{mg} \mathrm{dm}^{-3}\right)$; $\mathrm{K}$ (50 $\left.\mathrm{mg} \mathrm{dm}^{-3}\right)$;

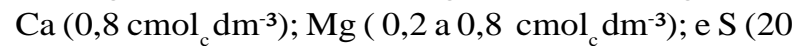
a $80 \mathrm{mg} \mathrm{dm}^{-3}$ ), dependendo do solo utilizado.

Todavia, recomendam-se novos estudos com os nutrientes $\mathrm{Ke} \mathrm{Ca}$, com doses inferiores às menores testadas, visto que, por este estudo, detectou-se que a melhor dose está entre os teores existentes originalmente no solo e a menor dose aplicada.

\section{AGRADECIMENTOS}

Os autores agradecem ao CNPq pela concessão de bolsa de estudo e de produtividade em pesquisa, e ao projeto PRODETAB 13-02/01, pelo financiamento do presente trabalho.

\section{REFERÊNCIAS}

ALVAREZ V., V. H. Equilíbrio de formas disponíveis de fósforo e enxofre em dois Latossolos de Minas Gerais. 1974. 125f. Dissertação (Mestrado em Solos e Nutrição de Plantas) - Universidade Federal de Viçosa, Viçosa, MG, 1974.

BALIERO, F. C.; OLIVEIRA, I. G.; DIAS, L. E. Formação de mudas de Acacia holosericea e Acacia auriculiformis :resposta a calagem, fósforo, potássio e enxofre. Revista Árvore, v. 25, n. 2, p. 183-191, 2001.

BERNARDINO, D. C. S. et al. Crescimento e qualidade de mudas de Anadenanthera macrocarpa (Benth.) brenan em resposta à saturação por bases do substrato. Revista Árvore., v.29, n.6, 2005. Disponível em: <http://www.scielo.br/ scielo.php?script $=$ sci_arttext\&pid=S0100$67622005000600004 \& \operatorname{lng}=$ pt\&nrm=iso $>$. Acesso em: 28 abr. 2007.

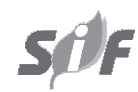

CARVALHO, P. E. R. Espécies arbóreas brasileiras. Brasília: Embrapa Informação Tecnológica; Colombo: Embrapa Florestas, 2003. 1039p.

CECONI, D. E. et al. Crescimento de mudas de açoita-cavalo (Luehea divaricata mart.) sob influência da adubação fosfatada. Revista Cerne, v. 12, n.3, p.292-299, 2006.

CRESTANA, C. S. M. et al. Sombreamento e adubação nitrogenada no crescimento de mudas de guarantã - Esenbeckia leiocarpa Engl. (Rutaceae). Revista do Instituto Florestal, v.7, n.2, p.115-123, 1995.

DIAS, L. E. et al. Formação de mudas de táxibranco (Sclerolobium paniculatum Voguel): Resposta a nitrogênio, potássio e enxofre. Revista Árvore, v.16, n.2, p.135-143, 1992.

DUBOC, E. et al. Nutrição do jatobá (Hymenaea coubaril L. var. stilbocarpa (Hayne)Lee et Lang).Cerne, Lavras, v.2, n.1, p. 31-47, 1996

EUCLYDES, R. F. Manual de utilização do programa SAEG (Sistema para analises estatísticas e genéticas). Viçosa, MG: Universidade Federal de Viçosa, 1997. 59p.

LORENZI, H. Árvores brasileiras. Nova Odessa: Plantarum, 2000. v.1. 352p.

MAFFEIS, A. R.; SILVEIRA, R. L. V A.; BRITO, J. O. Reflexos das deficiências de macronutrientes e boro no crescimento de plantas, produção e qualidade de óleo essencial em Eucalyptus citriodora. Scientia

Forestalis, n.57, p.87-98, 2000.

MARQUES, V. B. et al. Efeito de fontes e doses de nitrogênio sobre o crescimento inicial e qualidade de mudas de jacarandá-da-baía (Dalbergia nigra (Vell.) Fr. All. ex Benth.). Revista Árvore, v.30, n.5, 2006. Disponível em: <http://www.scielo.br/ scielo.php?script $=$ sci_arttext\&pid $=$ S010067622006000500006\&lng=pt\&nrm=iso >. Acesso em: 20 Maio de 2008.

MISSIO, E. L. et al . Exigências nutricionais da grápia ao fósforo e enxofre em Argissolo Vermelho distrófico arênico: efeito da adubação no crescimento. Ciencia Rural., v.34, n.4, 2004. Disponível em: <http:// www.scielo.br/scielo.php?script=sci_arttext\&pid=S0103$84782004000400013 \& \operatorname{lng}=$ pt\&nrm=iso $>$. Acesso em: 28 Abr 2007.

R. Árvore, Viçosa-MG, v.32, n.6, p.1029-1040, 2008 
NICOLOSO, F. T. et al. Nutrição mineral de mudas de grápia (Apuleia leiocarpa) em Argissolo Vermelho Distrófico arênico. (1) Efeito da adubação NPK no crescimento. Ciência Rural, v.31, n.6, p.1-8, 2001.

NOVAIS, R. F. et al. Calagem e adubação mineral na produção de mudas de eucalipto (Eucalyptus grandis W. Hill ex Maiden). I. Efeitos da calagem e dos nutrientes N, P e K. Revista Árvore, v.3, n.2, p.121-134, 1979.

OLIVEIRA, J. M. F. et al. Respostas de mudas de Angelim-pedra (Dinizia excelsa Ducke) a nitrogênio e fósforo. Pesquisa Agropecuária Brasileira, v.33, n.9, p.1-5, 1998.

PARON, M. E. et al. Crescimento da copaíba e guatambu em resposta a fungo micorrízico, superfosfato, nitrogênio e fumigação do solo. Cerne, v.2, n.2, p.15-30, 1996.

RENÓ, N. B. et al. Limitações nutricionais ao crescimento inicial de quatro espécies arbóreas nativas em Latossolo Vermelho-Amarelo. Pesquisa Agropecuária Brasileira, v.32, n.1, p.17-25, 1997.
RESENDE, M.; CURI, N.; SANTANA, D. P. Pedologia e fertilidade do solo: interações e aplicações. Brasília:Ministério da Educação; Lavras:ESAL; Piracicaba:POTAFOS, $1988.81 \mathrm{p}$.

SCHUMACHER, M. V.; CECONI, D. E.;

SANTANA, C. A. Influência de diferentes doses de fósforo no crescimento de mudas de angicovermelho (Parapiptadenia rigida (Bentham) Brenan). Revista Árvore, v.28, n. 1, 2004. Disponível em: <http://www.scielo.br/ scielo.php?script $=$ sci_arttext\&pid $=$ S0100$67622004000100019 \& \operatorname{lng}=\mathrm{pt} \& \mathrm{nrm}=\mathrm{iso}>$. Acesso em: 14 Jun 2007.

TURRENT, F. A. Uso de uma matriz mixta para la optimización de cinco a ocho factores controlables de la producción. Chapingo-México: Rama de Suelos, Colégio de Postgraduados, 1979. 65p. (Boletim Técnico, 6)

VENTURIN, N. et al. Adubação mineral do Angico-Amarelo (Peltophorum dubium (SPRENG.) TAUB.). Pesquisa Agropecuária Brasileira, v.34, n.3, p.441-448, 1999. 